\title{
Circular RNAs in body fluids as cancer biomarkers: the new frontier of liquid biopsies
}

Sumeng Wang ${ }^{1+}$, Ke Zhang ${ }^{1+}$, Shanyue Tan ${ }^{1+}$, Junyi Xin ${ }^{2,3}$, Qianyu Yuan ${ }^{4}$, Huanhuan Xu', Xian Xu', Qi Liang ${ }^{1}$, David C. Christiani ${ }^{4,5}$, Meilin Wang ${ }^{2,3^{*}}$, Lingxiang Liu ${ }^{1 *}$ and Mulong Du ${ }^{6^{*}}$

\begin{abstract}
Cancer is a leading cause of death worldwide, particularly because of its high mortality rate in patients who are diagnosed at late stages. Conventional biomarkers originating from blood are widely used for cancer diagnosis, but their low sensitivity and specificity limit their widespread application in cancer screening among the general population. Currently, emerging studies are exploiting novel, highly-accurate biomarkers in human body fluids that are obtainable through minimally invasive techniques, which is defined as liquid biopsy. Circular RNAs (circRNAs) are a newly discovered class of noncoding RNAs generated mainly by pre-mRNA splicing. Following the rapid development of high-throughput transcriptome analysis techniques, numerous circRNAs have been recognized to exist stably and at high levels in body fluids, including plasma, serum, exosomes, and urine. CircRNA expression patterns exhibit distinctly differences between patients with cancer and healthy controls, suggesting that circRNAs in body fluids potentially represent novel biomarkers for monitoring cancer development and progression. In this study, we summarized the expression of circRNAs in body fluids in a pan-cancer dataset and characterized their clinical applications in liquid biopsy for cancer diagnosis and prognosis. In addition, a user-friendly web interface was developed to visualize each circRNA in fluids (https://mulongdu.shinyapps.io/circrnas_in_fluids/).
\end{abstract}

Keywords: Circular RNA, Liquid biopsy, Cancer biomarker

\footnotetext{
* Correspondence: drdumulong@njmu.edu.cn; mwang@njmu.edu.cn; IIxlau@163.com

†Sumeng Wang, Ke Zhang and Shanyue Tan contributed equally to this work.

${ }^{2}$ Department of Environmental Genomics, Jiangsu Key Laboratory of Cancer Biomarkers, Prevention and Treatment, Collaborative Innovation Centre for Cancer Personalized Medicine, Nanjing Medical University, Nanjing, People's Republic of China

${ }^{1}$ Department of Oncology, The First Affiliated Hospital of Nanjing Medical University, 300 Guangzhou Road, Nanjing 210029, Jiangsu, People's Republic of China

${ }^{6}$ Department of Biostatistics, Centre for Global Health, School of Public Health, Nanjing Medical University, 101 Longmian Avenue, Nanjing 211166, Jiangsu, People's Republic of China

Full list of author information is available at the end of the article
}

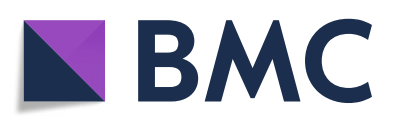

(- The Author(s). 2021 Open Access This article is licensed under a Creative Commons Attribution 4.0 International License, which permits use, sharing, adaptation, distribution and reproduction in any medium or format, as long as you give appropriate credit to the original author(s) and the source, provide a link to the Creative Commons licence, and indicate if changes were made. The images or other third party material in this article are included in the article's Creative Commons licence, unless indicated otherwise in a credit line to the material. If material is not included in the article's Creative Commons licence and your intended use is not permitted by statutory regulation or exceeds the permitted use, you will need to obtain permission directly from the copyright holder. To view a copy of this licence, visit http://creativecommons.org/licenses/by/4.0/. The Creative Commons Public Domain Dedication waiver (http://creativecommons.org/publicdomain/zero/1.0/) applies to the data made available in this article, unless otherwise stated in a credit line to the data. 


\section{Background}

Cancer is an important public health issue worldwide and the second leading cause of death in the United States [1]. Cancer arises from genetic alterations and dysregulated transcriptional programmes [2]. In recent years, cancer has imposed a tremendous burden on individuals, families, communities and health systems [3]. However, the early detection of cancer can help to minimize the cancer burden [4]. Over the past few decades, several blood-based biomarkers [e.g., carcinoembryonic antigen (CEA) and prostate-specific antigen (PSA)] have been used for the early detection of cancers, but the lack of sensitive and specific biomarkers has limited the early diagnosis and determination of the prognosis of many patients with cancer [4]. For example, PSA is present in both patients with prostate cancer and patients with benign prostate hyperplasia [5]. Thus, studies of biomarkers with high specificity and sensitivity are urgently needed.

Liquid biopsy is a non-invasive method that uses body fluids, such as blood, plasma, serum, urine, and gastric juice, to reflect the disease state [6]. Recently, substantial attention has been devoted to detecting and quantifying biomarkers, especially circular RNAs (circRNAs), in tumour biopsies [7]. Although the study on circRNAs is in its infancy, several studies have revealed their potential as valuable diagnostic and prognostic biomarkers for cancers [8]. CircRNAs are highly resistant to RNase activity because of the lack of $5^{\prime}$ and $3^{\prime}$ ends [9-11]. In addition, circRNAs are ideal candidates as liquid biopsy biomarkers, as they are often expressed in tissue- and developmental stage-specific manners, and are found in large quantities not only in tissues and cells but also in body fluids $[8,12]$.

In this review, we summarized the expression pattern of circRNAs in body fluids across pan-cancers and characterized their clinical applications in liquid biopsy. The receiver operating characteristic (ROC) curves and the corresponding area under the curve (AUC) values extracted from each analysis were used to describe the diagnostic value of the circRNAs. In addition, we developed a user-friendly web interface that enabled interested individuals to easily browse and search for diseaserelated circRNAs.

\section{Cancer biomarkers for liquid biopsies}

Liquid biopsy, a measurement of monitoring tumours in real-time, is a non-invasive biopsy method that has been investigated by scientists and oncologists for several years $[13,14]$. The aim of liquid biopsy is to identify and quantify the tumour-derived biological materials circulating in body fluids [15]. Several researchers have described the advantages of liquid biopsy compared with solid biopsy [16-18]. First, liquid biopsy detects the presence of a tumour at an earlier stage before it has been detected by radiological and imaging examinations $[16,19]$. Second, liquid biopsies may better characterize the whole tumour, not just a small area, which is a particularly important consideration for histologically heterogeneous tumours; in contrast, classical solid biopsies, which analyse only specific sections of malignancies, might ignore important molecular traits [16, 20]. Third, the possibility of repeated sampling via liquid biopsy allows more accurate and more dynamic monitoring of disease progression and modulation of the treatment strategy $[16,21]$. Moreover, liquid biopsy is a convenient way to monitor progression and recurrence early during patient follow-up [21, 22]. In conclusion, liquid biopsy may support the "gold standard" tissue biopsy or even replace tissue biopsy in the future.

\section{Biological characteristics of circRNAs}

When circRNAs were first discovered in the early 1970s, they were thought to be by-products of splicing without any valuable biological functions [23]. However, with the emergence of next-generation sequencing technology and the development of bioinformatics pipelines, numerous circRNAs have recently been discovered to be differentially expressed among patients with various cancers [7, 24]. During the process named back-splicing, circRNAs are generated from linear pre-messenger RNAs, and the $3^{\prime}$ and 5 ' ends are ligated to form a covalently closed, uninterrupted loop [24]. These closed-loop structures originate from the ligation of introns, exons, or both [7, 25]. The potential biogenetic and functional mechanisms of circRNAs are presented in Fig. 1.

Several studies have shown that most circRNAs contain miRNA response elements (MREs), functioning as miRNA sponges [26]. Moreover, circRNAs have been reported to increase the expression of innumerable mRNAs through an indirect mechanism [27]. CircRNAs can also bind RNA binding proteins (RBPs), such as MBL [28], SR proteins [29], and IGF2BP3 [7, 30]. In addition, circRNAs can be translated due to the presence of an internal ribosome entry site (IRES) in the sequence [31].

\section{CircRNAs in body fluids from a pan-cancer dataset as cancer biomarkers}

Recently, many circRNAs that function as oncogenes or tumour-suppressors have been discovered to be dysregulated in tumour specimens. Based on emerging evidence, circRNAs are associated with cancer progression and are involved in the malignant cellular behaviours [32-35]. As the shape of circRNAs is a covalently closed continuous loop, they have relatively stable framework in the eukaryotic transcriptome [36, 37]. Unlike linear RNAs, circRNAs are relatively stable and are not easily degraded by endonucleases [36, 38-40]. Here, we 


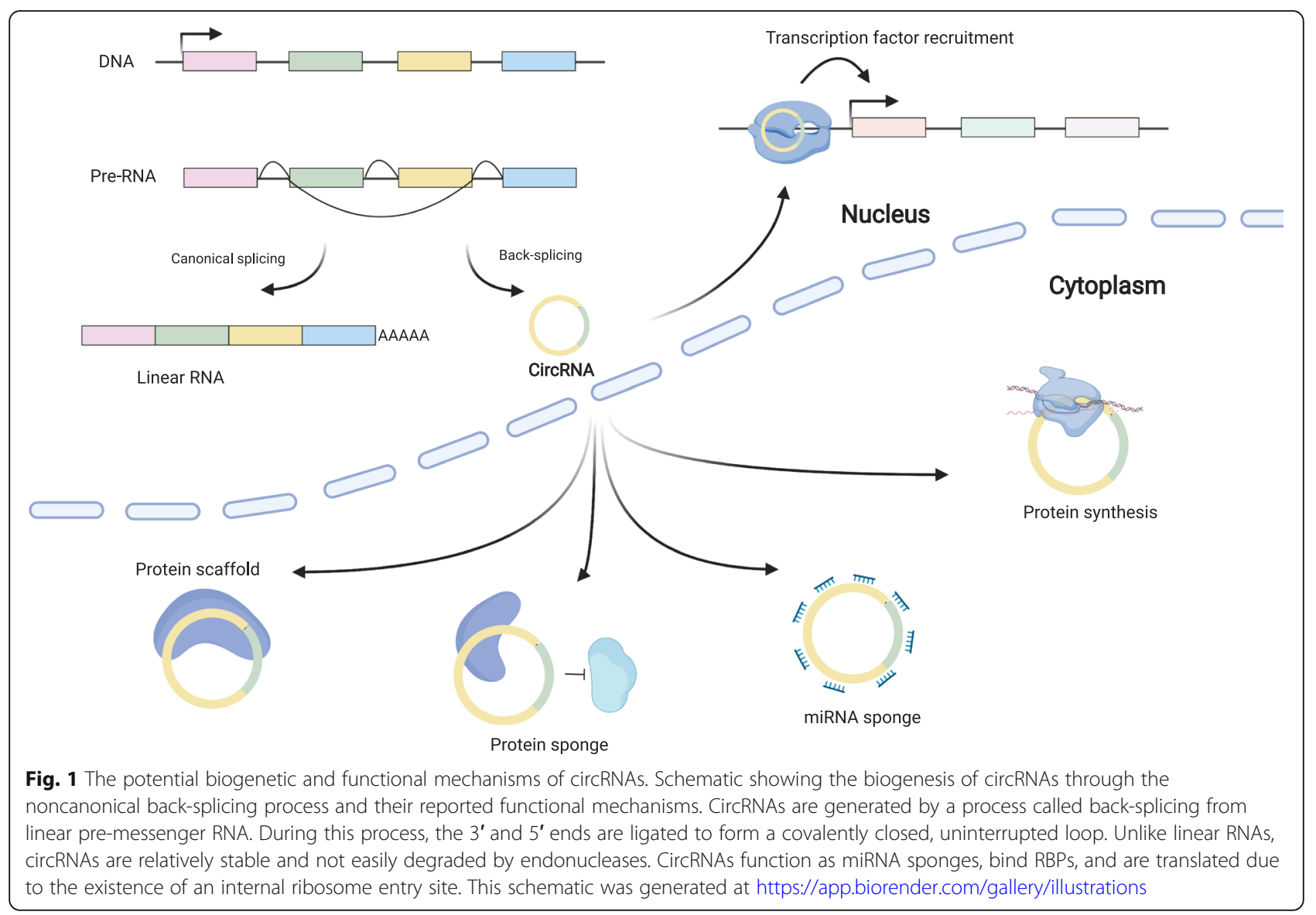

summarize recent studies on circRNAs in body fluids, including 112 differentially expressed circRNAs identified in various cancers from a search of PubMed for studies published up to 15 May, 2020 with the following key words: "circular RNA" OR "circular RNAs" OR "circRNA" OR "circRNAs" AND "cancer" AND ("liquid biopsies" OR "biomarker" OR "non-invasive"). Detailed information about these 112 circRNAs is presented in Supplementary Figure 1 and Supplementary Table 1 . Figure 2 shows the circulating circRNAs identified in the ten most common cancers. In addition, a new intuitive web interface for users to explore and analyse circRNAs was developed using the shiny package in $\mathrm{R}$ (Fig. 3), which is easily accessed at https://mulongdu.shinyapps. io/circrnas_in_fluids/.

\section{Lung cancer}

Lung cancer is the leading cause of cancer-related morbidity and mortality worldwide, of which non-small cell lung cancer (NSCLC) accounts for more than $80 \%$ of lung cancer cases [1, 41]. CircFARSA, derived from exons 5-7 of FARSA gene, was present at higher levels in plasma from patients than from healthy controls, indicating that plasma circFARSA can act as a potential non-invasive diagnostic biomarker for lung cancer [42].
Tan et al. observed that the suppression of cell migration and invasion after silencing F-circEA-2a [43]. Zhu et al. identified plasma hsa_circ_0013958 as a functional diagnostic and prognostic biomarker, because its presence in plasma from patients with lung cancer positively correlated with the TNM stage and lymphatic metastasis, with an AUC of 0.815 , and it could promote cell proliferation and invasion as well as inhibit apoptosis [32]. Additionally, serum exosomal circRNAs FECR1 and FECR2 played important roles in lung cancer progression [44].

\section{Breast cancer}

Breast cancer is the second most frequently diagnosed cancer and the most common cancer in females [1, 41]. The plasma level of hsa_circ_0001785 was closely correlated with the histological grade, TNM stage, and distant metastasis of breast cancer, with higher diagnostic value $($ AUC $=0.784)$ than both CEA $($ AUC $=0.562)$ and carbohydrate antigen 153 (CA153) (AUC $=0.629)$ [45]. In addition, hsa_circ_0068033 and hsa_circ_0108942 in plasma had potential diagnostic value in the clinic [45]. Wang et al. reported the significant overexpression of hsa_circ_0020707, hsa_circ_0064923, hsa_circ_0104852, hsa_circ_0087064, and hsa_circ_0009634 in the serum from patients with breast cancer and positive 


\begin{tabular}{|c|c|c|}
\hline $\begin{array}{l}\text { Esophageal Squamous Cell Carcinoma: } \\
\text { circ-SMAD7 } \\
\text { circ-TTC17 } \\
\text { hsa_circ_0001946 } \\
\text { hsa_circ_0043603 } \\
\text { hsa_circ_0062459 } \\
\text { circGSK33 } \\
\text { hsa_circ_0004771 } \\
\text { circ-SLC7A5 } \\
\text { Lung Cancer: } \\
\text { F-circEA-2a } \\
\text { FECR1 } \\
\text { FECR2 } \\
\text { Circ-FARSA } \\
\text { hsa_circ_0013958 } \\
\text { hsa_circRNA_0056616 } \\
\text { Hepatocellular Carcinoma: } \\
\text { circ-ITCH } \\
\text { Circ-ZEB1.33 } \\
\text { hsa_circ_0027089 } \\
\text { hsa_circ_0000976 } \\
\text { hsa_circ_0007750 } \\
\text { hsa_circ_0139897 } \\
\text { circSMARCA5 } \\
\text { circ-DB } \\
\text { circ_0000798 } \\
\text { hsa_circ_0003998 } \\
\text { hsa_circ_0008043 } \\
\text { hsa_circ_0003731 } \\
\text { hsa_circ_0088030 } \\
\text { hsa_circ_0064428 } \\
\text { circ-ADD3 } \\
\text { circ-0051443 } \\
\text { circRNA_101237 }\end{array}$ & $\begin{array}{l}\text { Gastric Cancer: } \\
\text { hsa_circ_0000190 } \\
\text { hsa_circ_00001649 } \\
\text { hsa_circ_0006633 } \\
\text { hsa_circ_0000520 } \\
\text { Circ-SFMBT2 } \\
\text { hsa_circ_0000745 } \\
\text { hsa_circ_0061276 } \\
\text { hsa_circ_0001017 } \\
\text { circ-KIAA1244 } \\
\text { circ_SPECC1 } \\
\text { hsa_circ_0065149 } \\
\text { circSMARCA5 } \\
\text { hsa_circ_0000467 } \\
\text { circPSMC3 } \\
\text { ciRS-133 } \\
\text { hsa_circ_0000181 } \\
\text { hsa_circ_0021087 } \\
\text { hsa_circ_0005051 } \\
\text { hsa_circ_0006848 } \\
\text { hsa_circ_0010882 } \\
\text { circ-RanGAP1 } \\
\text { hsa_circ_0000419 } \\
\text { hsa_circ_002059 } \\
\text { Colorectal Cancer: } \\
\text { hsa_circ_0000338 } \\
\text { circZNF609 } \\
\text { circ-CCDC66 } \\
\text { circ-ABCC1 } \\
\text { circ-STIL } \\
\text { hsa_circ_0004771 } \\
\text { hsa_circ_0001649 } \\
\text { hsa_circ_0004585 } \\
\text { Circ-VAPA } \\
\text { circ-KLHDC10 } \\
\text { hsa_circ_0082182 } \\
\text { hsa_circ_0000370 } \\
\text { hsa_circ_0035445 }\end{array}$ & $\begin{array}{l}\text { Breast Cancer: } \\
\text { hsa_circ_0068033 } \\
\text { hsa_circ_0108942 } \\
\text { hsa_circ_0001785 } \\
\text { hsa_circ_0020707 } \\
\text { hsa_circ_0064923 } \\
\text { hsa_circ_0104852 } \\
\text { hsa_circ_0087064 } \\
\text { hsa_circ_000963 } \\
\text { hsa_circ_0069094 } \\
\text { hsa_circ_0079876 } \\
\text { hsa_circ_0017650 } \\
\text { hsa_circ_0017536 } \\
\text { circ_0007255 } \\
\text { Bladder Cancer: } \\
\text { hsa_circ_0003221 } \\
\text { hsa_circ_0000285 } \\
\text { CircCEP128 } \\
\text { circPRMT5 } \\
\text { Prostate Cancer: } \\
\text { circ_0044516 } \\
\text { circAR3 } \\
\text { circFOXO3 } \\
\text { Thyroid Cancer: } \\
\text { hsa-circ-020135 } \\
\text { hsa-circ-007293 } \\
\text { hsa-circ-031752 } \\
\text { circFNDC3B } \\
\text { Ovarian Cancer: } \\
\text { circ_0044516 } \\
\text { circAR3 } \\
\text { circFOX03 } \\
\text { Cervical Cancer: } \\
\text { circFox03a }\end{array}$ \\
\hline \multicolumn{3}{|c|}{$\begin{array}{l}\text { Fig. } 2 \text { CircRNAs identified in patients with the } 10 \text { most common cancers. The ten most common cancers are shown according to "cancer } \\
\text { statistics in } 2020 \text { "[1], including lung cancer, breast cancer, colorectal cancer, prostate cancer, gastric cancer, hepatocellular carcinoma, esophageal } \\
\text { squamous cell carcinoma, cervical cancer, thyroid cancer, and bladder cancer. Ovarian cancer-related circRNAs are listed in this figure because of } \\
\text { its increasing morbidity and mortality rates in females. This figure was generated at https://app.biorender.com/gallery/lllustrations }\end{array}$} \\
\hline
\end{tabular}

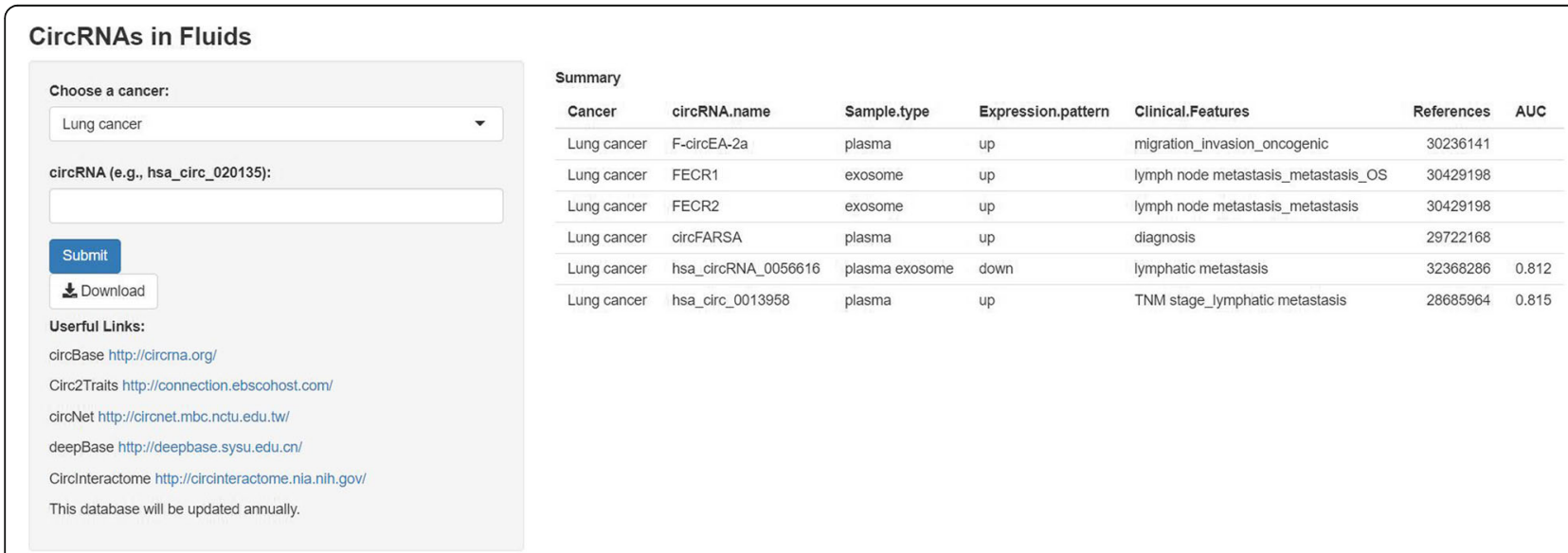

Fig. 3 A user-friendly interface to visualize the database of circRNAs in body fluids. The shiny package in R was utilized to develop this interface, and this interface is accessible in a user-friendly format at https://mulongdu.shinyapps.io/circrnas_in_fluids/. Moreover, some useful links are listed in this interface and users can click them to obtain more information. More detailed information can be acquired by navigating to this website or clicking on the hyperlink in the electronic version of this manuscript 
correlations with carcinogenesis and progression [46]. Additionally, Jia et al. documented higher levels of circ 0007255 in serum from patients compared to serum from healthy controls [47].

\section{Colorectal cancer}

Colorectal cancer is the third most common cancer worldwide [1, 41]. Three circRNAs (circ-CCDC66, circ$\mathrm{ABCC} 1$, and circ-STIL) were significantly downregulated in plasma from patients with colorectal cancer compared to healthy controls, and all of these circRNAs presented higher AUC values than those of commonly used cancer biomarkers, such as CEA and carbohydrate antigen 19-9 (CA19-9) [48]. Exosomal hsa_circ_0004771 and circKLHDC10 were significantly upregulated in patients with colorectal cancer, suggesting that they may represent potentially novel diagnostic biomarkers for colorectal cancer $[49,50]$. Tian et al. discovered that hsa_circ 0004585 was positively related to tumour size and involved in colorectal cancer carcinogenesis and metastasis; moreover, hsa_circ_0004585 derived from peripheral samples had an AUC of 0.707, with a sensitivity and specificity of 0.908 and 0.408 , respectively, indicating its clinical importance in diagnosing colorectal cancer [34]. A set of three circRNAs (hsa_circ_0000370, hsa_circ 0082812, and hsa_circ_0035445) were found to be dysregulated in colorectal cancer plasma, with AUCs of $0.815,0.737$, and 0.703 , respectively [51]. Li et al. verified that the increased plasma circVAPA (hsa_circ_0006990) levels could exert oncogenic effects by sponging miR101 to affect colorectal cancer development [52]. The expression of circZNF609 was downregulated in the serum from patients with colorectal cancer [53]; and hsa_circ_0001649 showed a close relation to pathological differentiation, with an AUC of 0.857 [54].

\section{Prostate cancer}

Prostate cancer is the fourth most frequently diagnosed cancer, with a high cancer-related mortality rate worldwide [1, 41]. Exosomal circ_0044516 was significantly upregulated in patients with prostate cancer and regulated the proliferation and metastasis of cancer cells by modulating miR-29a-3p expression [55]. Kong et al. reported the upregulation of circFOXO3 (hsa_circ 0006404), which was derived from exon 2 of the forkhead box $\mathrm{O} 3$ (FOXO3) gene, in serum from patients with prostate cancer; circFOXO3 exhibited oncogenic activity by altering the cell cycle and apoptosis in the process of sponging miR-29a-3p to regulate SLC25A15 expression [33].

\section{Gastric cancer}

Gastric cancer is the fifth most frequently diagnosed malignancy and the third leading cause of cancer-related death worldwide [1, 41]. Twenty-three circRNAs have been discovered to be dysregulated in patients with gastric cancer, and 18 of which were downregulated and 5 were upregulated; detailed information is presented in Supplementary Table 1. Plasma hsa_circ_0000419 level was significantly associated with advanced tumour stage, lymphatic and distal metastasis, as well as venous and perineural invasion of gastric cancer, serving as a prognostic indicator and tumour suppressor [35]. The plasma level of hsa_circ_0000190 was decreased in patients with gastric cancer, with a high AUC of 0.600 , and was associated with the tumour diameter, TNM stage, lymphatic metastasis, distal metastasis, and CA19-9 level, indicating that hsa_circ_0000190 could serve as a novel noninvasive diagnostic biomarker [56]. The low plasma exosomal hsa_circ_0065149 level in patients with early stage gastric cancer suggested that hsa_circ_0065149 would be a useful indicator for the early screening of gastric cancer [57]. Rong et al. observed significant correlations between a lower expression level of circPSMC3 in patients with gastric cancer and a higher TNM stage and shorter overall survival (OS) time; and circPSMC3 could participate in the progression of gastric cancer by sponging miRNA-296-5p to regulate the expression of Phosphatase and Tensin Homolog (PTEN), providing new insights into the treatment of gastric cancer [58]. Hsa_circ 0000467 was noticeably overexpressed in plasma from patients with gastric cancer, with an AUC of 0.790, a value that was higher than those of traditional cancer biomarkers, such as CEA and carbohydrate antigen 724 (CA-724). In terms of biological function, knockdown of hsa_circ_0000467 markedly inhibited the proliferation, invasion, and migration of gastric cancer cells, indicating that hsa_circ_0000467 could play a functional role as a non-invasive diagnostic and prognostic biomarker for gastric cancer [59].

\section{Hepatocellular carcinoma}

Hepatocellular carcinoma (HCC), the sixth most common cause of cancer-related death worldwide, accounts for approximately $80 \%$ of primary liver cancers [1, 41]. Chen et al. discovered a significantly lower expression level of circ_0051443 in plasma exosomes from patients than that from healthy controls, serving as a predictive and diagnostic biomarker, and it could suppress malignant biological behaviours by regulating apoptosis, proliferation, and cell cycle arrest [60]. In addition, the plasma level of hsa_circ_0027089, hsa_circ_0000976, hsa_circ_0007750, and hsa_circ_0139897 have been used to distinguish patients with $\mathrm{HCC}$ from healthy controls $[61,62]$. The plasma level of hsa_circ_0003998 in healthy controls was significantly lower than that in patients with HCC, with an AUC value of 0.892 alone and 0.947 in combination with alpha fetoprotein (AFP). In 
addition, patients with lower plasma hsa_circ_0003998 levels experienced a prolonged OS compared with patients with higher levels, indicating that hsa_circ 0003998 represented a potential diagnostic and prognostic biomarker for HCC [63]. A lower expression level of circSMARCA5 has been observed in plasma from patients with $\mathrm{HCC}$ than that from healthy controls with high discriminatory accuracy (AUC $=0.938$ ), serving as a biomarker for distinguishing patients with HCC from healthy controls [64]. Weng et al. reported correlations between plasma hsa_circ_0064428 and HCC survival, tumour size, and metastasis, suggesting a role as a prognostic biomarker for HCC [65]. Serum circ-ZEB1.33 was associated with the TNM stage and prognosis, and this circRNA functioned as a miR-200a-3p sponge to upregulate CDK6 [66]. Moreover, low-expressed circ-ITCH [67] and circADD3 [68], as well as high-expressed circRNAs (hsa_circ 0008043 [69], hsa_circ_0003731 [69], hsa_circ_0088030 [69], circ-DB [70], and circ_0000798 [71]), played potential prognostic roles in patients with HCC.

\section{Esophageal squamous cell carcinoma}

Esophageal squamous cell carcinoma (ESCC) has high incidence and mortality rates, and ranks the seventh among the most frequently diagnosed malignancies worldwide [1, 41]. Plasma levels of hsa_circ_00021946 and hsa_circ_0043603 detected were used as diagnostic biomarkers [72]. Plasma levels of circ-SMAD7 were significantly lower in patients with ESCC than that in normal controls and correlated with the TNM stage and lymphatic metastasis, serving as a novel diagnostic biomarker for ESCC [73]. Wang et al. demonstrated that upregulation of circ-TTC17 in patient plasma was correlated with the proliferation and migration of ESCC cells, proving that circ-TTC17 had potential prognostic value for ESCC [74]. In addition, the expression level of circGSK3 $\beta$ could be as a novel non-invasive biomarker for detecting ESCC and early stage ESCC with AUCs of 0.782 and 0.793 , respectively [75]. Upregulation of hsa circ_0004771 in plasma was associated with a heavier tumour burden and poor prognosis, and hsa_circ 0004771 knock down could inhibit ESCC cell proliferation via the miR-339-5p/CDC25A axis [76]. Wang et al. discovered a correlation between high plasma levels of circ-SLC7A5 in patients with a high TNM stage, suggesting a role as a prognostic biomarker [77].

\section{Cervical cancer}

Cervical cancer ranks eighth among the most frequently diagnosed cancers worldwide, and it is the fourth most frequent and important cause of cancer-related mortality in women $[1,41]$. CircFoxO3a, which was downregulated in patient serum, was associated with deeper stromal invasion and positive lymph node metastasis; in addition, patients with higher expression levels of serum circFoxO3a would have better prognoses [78].

\section{Thyroid cancer}

Thyroid cancer is the ninth most common cancer worldwide $[1,41]$. Wu et al. documented the high expression of circFNDC3B in serum exosomes from patients with thyroid cancer, and this circRNA modulated thyroid cancer progression via the miR-1178/TLR4 pathway [79].

\section{Bladder cancer}

Bladder cancer ranks tenth among the commonly diagnosed cancers, with a high recurrence rate worldwide $[1,41]$. Blood hsa_circ_0003221 was significantly associated with clinicopathological characteristics, including poor differentiation, lymph node metastasis, and high $\mathrm{T}$ stage; and was involved in the inhibition of cell proliferation and migration [80]. Chi et al. discovered the low expression level of hsa_circ 0000285 in serum from patients with bladder cancer was associated with tumour size, tumour differentiation, distant metastasis, and TNM stage, indicating that hsa_circ 0000285 represented a novel prognostic biomarker for bladder cancer [81]. In addition, circPRMT5 was upregulated in serum and urinary exosomes from patients with bladder cancer and exhibited a strong correlation with tumour metastasis [82].

\section{Ovarian cancer}

Recently, the morbidity and mortality rates of ovarian cancer have increased annually [1, 41]. Hu et al. observed the downregulation of circBNC2 in plasma samples from patients with ovarian cancer compared with that from healthy controls [83]. The high serum circSETDB1 levels identified in patients with ovarian cancer were significantly increased in patients with primary chemoresistance, which could distinguish patients with ovarian cancer from healthy controls and patients with primary chemoresistance from those with primary chemosensitivity; these findings identified a role for serum circSETDB1 levels as a diagnostic and prognostic biomarker [84]. In addition, Cdr1as, which was involved in the mechanism regulating the miR-1270/SCAI signalling pathway, was expressed at low levels in serum exosomes from patients with cisplatin resistance [85].

\section{Other common tumours}

In addition to these top cancers, circRNAs are also involved in the tumorigenesis of many other cancers. For example, hsa_circ_0081001 was overexpressed in serum from patients with osteosarcoma and exhibited a better diagnostic and prognostic value than either alkaline phosphatase (ALP) or lactate dehydrogenase (LDH) [86]; increased serum hsa_circ_0000885 levels showed a similar diagnostic value [87]. Higher plasma levels of circ- 
LDLRAD3 have been detected in patients with pancreatic cancer than those in healthy controls, and were associated with venous invasion, lymphatic invasion, and metastasis, with an AUC of 0.670 alone and a higher AUC of 0.870 in combination with CA19-9 [88]. In patients with nasopharyngeal carcinoma, serum levels of circRNA_0000285 were increased and significantly correlated with the differentiation grade, tumour size, lymph node metastasis, distal metastasis, and TNM stage, indicating an independent prognostic indicator for nasopharyngeal carcinoma [89]. In addition, hsa_circ_ 0109046 and hsa_circ_0002577 were upregulated in the serum of patients with endometrial cancer [90], and exosomal circ-0000284 was overexpressed in patients with cholangiocarcinoma [91].

\section{Conclusions and future perspectives}

In this review, we comprehensively summarized clinically valuable circRNAs in body fluids as diagnostic and prognostic cancer biomarkers.

Due to its high morbidity and mortality rates, cancer is a major threat to human health [2]. Accounting for the stateof-the-art detection technologies, including next-generation sequencing, an increasing number of circRNAs that are differentially expressed in different clinical stages have been examined and quantified [7, 24]. Compared to conventional cancer biomarkers (e.g. PSA and CEA), circRNAs have higher sensitivity and specificity in diagnosis and prognosis, and play an increasingly important role in the oncogenesis and progression of diverse tumours $[48,59]$.

CircRNAs have various biological mechanisms, including the mainly reported function of miRNA sponges, which could increase the complexity of the competing endogenous RNA (ceRNA) network. In addition, studies exploring the other biological functions of circRNAs in tumours, such as their roles as gene regulators, should not be ignored [92-95].

Currently, many studies have identified that circRNAs as potentially novel diagnostic and prognostic biomarkers for multiple tumours. Although the detection of circRNAs has mainly been conducted in tissues and cell lines, more studies have focused on circRNAs detection using less invasive and more accessible methods, such as liquid biopsy $[6,15,16]$. Moreover, combinations of circRNAs with traditional cancer biomarkers may exhibit higher diagnostic or prognostic accuracy than single traditional biomarkers [63].

Different from previous reviews about circRNAmiRNA-mRNA axis that have mainly described biological functions in tumour tissues or cells, we reviewed the expression of circRNAs in body fluids from patients with various tumours and summarized their diagnostic and prognostic values as non-invasive biomarkers in clinical applications; furthermore, we made a user- friendly interface for readers to obtain the corresponding data [8, 96-98].

We have to acknowledge that difficulties and challenges of circRNAs existed in clinical applications [22]. First, circRNAs are difficult to accurately detect and recognize in body fluids due to their low abundance [99]. Second, the detection methods must to be improved to enable increased precision in detecting circRNAs [99]. Thus, we should improve advanced technologies and experimental approaches to apply circRNAs in clinical practice.

Research into circRNAs as cancer biomarkers is still in its infancy, and little is known about their mechanisms in tumorigenesis and tumour progression. Because of the substantial improvements that are ongoing, we propose that most of these limitations will be overcome and that more circRNAs will be detected and applied in the clinic in the future.

\section{Supplementary Information}

The online version contains supplementary material available at https://doi. org/10.1186/s12943-020-01298-z.

Additional file 1: Figure S1. The total number of circRNAs in the pancancer dataset. Supplementary Table 1. Summary of the expression pattern and sample type of circRNAs involved in pan-cancer

\section{Abbreviations \\ circRNAs: circular RNAs; PSA: Prostate specific antigen; ROC curve: Receiver operating characteristic curve; AUC: The area under ROC curve; MREs: miRNA response elements; RBPs: RNA binding proteins; IRES: Internal ribosome entry site; NSCLC: Non-small cell lung cancer; CEA: Carcinoembryonic antigen; CA19-9: Carbohydrate antigen 19-9; CA153: Carbohydrate antigen 153; FOXO3: Forkhead box O3; PTEN: Phosphatase and Tensin Homolog; CA- 724: Carbohydrate antigen 724; HCC: Hepatocellular carcinoma; AFP: Alpha fetoprotein; ESCC: Esophageal squamous cell carcinoma; ALP: Alkaline phosphatase; LDH: Lactate dehydrogenase; ceRNA: competing endogenous RNA; OS: Overall survival}

\section{Acknowledgements}

Not applicable.

\section{Authors' contributions}

Lingxiang Liu, Mulong Du, Meilin Wang researched on the background of the study. Mulong Du supervised the project. Sumeng Wang, Ke Zhang, Huanhuan Xu, Xian Xu and Qi Liang made figures and tables. Sumeng Wang, Ke Zhang and Shanyue Tan drafted the initial manuscript. Ke Zhang, Junyi Xin, Sumeng Wang constructed the circRNA database. Junyi Xin set up the website. David C Christiani commented on the manuscript. Qianyu Yuan revised and polished the manuscript. All authors read and approved the final manuscript.

\section{Funding}

This study was partly supported by National Natural Science Foundation of China (81703307), the Natural Science Foundation of Jiangsu Province (BK20181371) and the Priority Academic Program Development of Jiangsu Higher Education Institutions (Public Health and Preventive Medicine).

\section{Availability of data and materials}

All data were available and accessible in web (https://mulongdu.shinyapps. io/circrnas_in_fluids/).

Ethics approval and consent to participate

Not applicable. 


\section{Consent for publication}

All authors give consent for the publication of manuscript in Molecular Cancer.

\section{Competing interests}

The authors declare that they have no competing interests.

\section{Author details}

'Department of Oncology, The First Affiliated Hospital of Nanjing Medical University, 300 Guangzhou Road, Nanjing 210029, Jiangsu, People's Republic of China. ${ }^{2}$ Department of Environmental Genomics, Jiangsu Key Laboratory of Cancer Biomarkers, Prevention and Treatment, Collaborative Innovation Centre for Cancer Personalized Medicine, Nanjing Medical University, Nanjing, People's Republic of China. ${ }^{3}$ Department of Genetic Toxicology, The Key Laboratory of Modern Toxicology of Ministry of Education, Centre for Global Health, School of Public Health, Nanjing Medical University, 101 Longmian Avenue, Nanjing 211166, Jiangsu, People's Republic of China. ${ }^{4}$ Departments of Environmental Health, Harvard T.H. Chan School of Public Health, 677 Huntington Avenue, Boston, MA 02115, USA. ${ }^{5}$ Department of Medicine, Massachusetts General Hospital, 55 Fruit Street, Boston, MA 02114, USA. ${ }^{6}$ Department of Biostatistics, Centre for Global Health, School of Public Health, Nanjing Medical University, 101 Longmian Avenue, Nanjing 211166, Jiangsu, People's Republic of China.

Received: 25 June 2020 Accepted: 21 December 2020

Published online: 11 January 2021

\section{References}

1. Siegel RL, Miller KD, Jemal A. Cancer statistics, 2020. CA Cancer J Clin. 2020; 70(1):7-30.

2. Bradner JE, Hnisz D, Young RA. Transcriptional addiction in Cancer. Cell. 2017;168(4):629-43.

3. Collaborators GBDRF. Global, regional, and national comparative risk assessment of 79 behavioural, environmental and occupational, and metabolic risks or clusters of risks, 1990-2015: a systematic analysis for the global burden of disease study 2015. Lancet. 2016;388(10053):1659-724.

4. Schiffman JD, Fisher PG, Gibbs P. Early detection of cancer: past, present, and future. Am Soc Clin Oncol Educ Book. 2015:57-65. https://doi.org/10. 14694/EdBook_AM.2015.35.57.

5. Filella X, Foj L. Prostate Cancer Detection and Prognosis: From Prostate Specific Antigen (PSA) to Exosomal Biomarkers. Int J Mol Sci. 2016;17(11): 1784.

6. Reimers N, Pantel K. Liquid biopsy: novel technologies and clinical applications. Clin Chem Lab Med. 2019;57(3):312-6.

7. de Fraipont F, Gazzeri S, Cho WC, Eymin B. Circular RNAs and RNA splice variants as biomarkers for prognosis and therapeutic response in the liquid biopsies of lung Cancer patients. Front Genet. 2019;10:390.

8. Arnaiz E, Sole C, Manterola L, Iparraguirre L, Otaegui D, Lawrie CH. CircRNAs and cancer: biomarkers and master regulators. Semin Cancer Biol. 2019;58: 90-9.

9. Memczak S, Jens M, Elefsinioti A, Torti F, Krueger J, Rybak A, et al. Circular RNAs are a large class of animal RNAs with regulatory potency. Nature. 2013:495(7441):333-8.

10. Li Z, Huang C, Bao C, Chen L, Lin M, Wang X, et al. Exon-intron circular RNAs regulate transcription in the nucleus. Nat Struct Mol Biol. 2015;22(3): 256-64.

11. Salzman J, Chen RE, Olsen MN, Wang PL, Brown PO. Cell-type specific features of circular RNA expression. PLoS Genet. 2013;9(9):e1003777.

12. Wilusz JE. A 360 degrees view of circular RNAs: from biogenesis to functions. Wiley Interdiscip Rev RNA. 2018;9(4):e1478.

13. Diaz LA Jr, Bardelli A. Liquid biopsies: genotyping circulating tumor DNA. J Clin Oncol. 2014;32(6):579-86

14. Karachaliou N, Mayo-de-Las-Casas C, Molina-Vila MA, Rosell R. Real-time liquid biopsies become a reality in cancer treatment. Ann Transl Med. 2015; 3(3):36.

15. Aghamir SMK, Heshmat R, Ebrahimi M, Khatami F. Liquid biopsy: the unique test for chasing the genetics of solid tumors. Epigenet Insights. 2020;13: 2516865720904052.

16. Mader S, Pantel K. Liquid biopsy: current status and future perspectives. Oncol Res Treat. 2017;40(7-8):404-8.
17. Mathai RA, Vidya RVS, Reddy BS, Thomas L, Udupa K, Kolesar J, et al. Potential Utility of Liquid Biopsy as a Diagnostic and Prognostic Tool for the Assessment of Solid Tumors: Implications in the Precision Oncology. J Clin Med. 2019;8(3):373.

18. Hench IB, Hench J, Tolnay M. Liquid Biopsy in Clinical Management of Breast, Lung, and Colorectal Cancer. Front Med (Lausanne). 2018;5:9.

19. Krishnamurthy N, Spencer E, Torkamani A, Nicholson L. Liquid Biopsies for Cancer: Coming to a Patient near You. J Clin Med. 2017;6(1):3.

20. Chang Y, Tolani B, Nie X, Zhi X, Hu M, He B. Review of the clinical applications and technological advances of circulating tumor DNA in cancer monitoring. Ther Clin Risk Manag. 2017;13:1363-74.

21. Heitzer E, Haque IS, Roberts CES, Speicher MR. Current and future perspectives of liquid biopsies in genomics-driven oncology. Nat Rev Genet. 2019;20(2):71-88.

22. Pardini B, Sabo AA, Birolo G, Calin GA. Noncoding RNAs in Extracellular Fluids as Cancer Biomarkers: The New Frontier of Liquid Biopsies. Cancers (Basel). 2019;11(8):1170.

23. Hsu MT, Coca-Prados M. Electron microscopic evidence for the circular form of RNA in the cytoplasm of eukaryotic cells. Nature. 1979;280(5720):339-40.

24. Dragomir M, Calin GA. Circular RNAs in Cancer - lessons learned from microRNAs. Front Oncol. 2018:8:179.

25. Wang J, Zhu M, Pan J, Chen C, Xia S, Song Y. Circular RNAs: a rising star in respiratory diseases. Respir Res. 2019;20(1):3.

26. Guo JU, Agarwal V, Guo H, Bartel DP. Expanded identification and characterization of mammalian circular RNAs. Genome Biol. 2014;15(7):409.

27. Hansen TB, Jensen TI, Clausen BH, Bramsen JB, Finsen B, Damgaard CK, et al. Natural RNA circles function as efficient microRNA sponges. Nature. 2013; 495(7441):384-8.

28. Ashwal-Fluss R, Meyer M, Pamudurti NR, Ivanov A, Bartok O, Hanan M, et al. circRNA biogenesis competes with pre-mRNA splicing. Mol Cell. 2014;56(1):55-66.

29. Barbagallo D, Caponnetto A, Cirnigliaro M, Brex D, Barbagallo C, D'Angeli F, et al. CircSMARCA5 Inhibits Migration of Glioblastoma Multiforme Cells by Regulating a Molecular Axis Involving Splicing Factors SRSF1/SRSF3/PTB. Int J Mol Sci. 2018;19(2):480.

30. Schneider T, Hung LH, Schreiner S, Starke S, Eckhof H, Rossbach O, et al. CircRNA-protein complexes: IMP3 protein component defines subfamily of circRNPs. Sci Rep. 2016;6:31313.

31. Pamudurti NR, Bartok O, Jens M, Ashwal-Fluss R, Stottmeister C, Ruhe L, et al. Translation of CircRNAs. Mol Cell. 2017;66(1):9-21 e7.

32. Zhu X, Wang X, Wei S, Chen Y, Chen Y, Fan X, et al. hsa_circ_0013958: a circular RNA and potential novel biomarker for lung adenocarcinoma. FEBS J. 2017;284(14):2170-82.

33. Kong Z, Wan X, Lu Y, Zhang Y, Huang Y, Xu Y, et al. Circular RNA circFOXO3 promotes prostate cancer progression through sponging miR-29a-3p. J Cell Mol Med. 2020;24(1):799-813.

34. Tian J, Xi X, Wang J, Yu J, Huang Q, Ma R, et al. CircRNA hsa_circ_0004585 as a potential biomarker for colorectal cancer. Cancer Manag Res. 2019;11: 5413-23.

35. Tao X, Shao Y, Lu R, Ye Q, Xiao B, Ye G, et al. Clinical significance of hsa_ circ_0000419 in gastric cancer screening and prognosis estimation. Pathol Res Pract. 2020;216(1):152763.

36. Li P, Chen S, Chen H, Mo X, Li T, Shao Y, et al. Using circular RNA as a novel type of biomarker in the screening of gastric cancer. Clin Chim Acta. 2015; 444:132-6.

37. Chen LL, Yang L. Regulation of circRNA biogenesis. RNA Biol. 2015;12(4): 381-8.

38. Hentze MW, Preiss T. Circular RNAs: splicing's enigma variations. EMBO J. 2013;32(7):923-5.

39. Jeck WR, Sorrentino JA, Wang K, Slevin MK, Burd CE, Liu J, et al. Circular RNAs are abundant, conserved, and associated with ALU repeats. Rna. 2013; 19(2):141-57.

40. Suzuki H, Zuo Y, Wang J, Zhang MQ, Malhotra A, Mayeda A. Characterization of RNase R-digested cellular RNA source that consists of lariat and circular RNAs from pre-mRNA splicing. Nucleic Acids Res. 2006; 34(8):e63.

41. Bray F, Ferlay J, Soerjomataram I, Siegel RL, Torre LA, Jemal A. Global cancer statistics 2018: GLOBOCAN estimates of incidence and mortality worldwide for 36 cancers in 185 countries. CA Cancer J Clin. 2018;68(6):394-424.

42. Hang D, Zhou J, Qin N, Zhou W, Ma H, Jin G, et al. A novel plasma circular RNA circFARSA is a potential biomarker for non-small cell lung cancer. Cancer Med. 2018;7(6):2783-91. 
43. Tan S, Sun D, Pu W, Gou Q, Guo C, Gong Y, et al. Circular RNA F-circEA-2a derived from EML4-ALK fusion gene promotes cell migration and invasion in non-small cell lung cancer. Mol Cancer. 2018;17(1):138.

44. Li L, Li W, Chen N, Zhao H, Xu G, Zhao Y, et al. FLI1 Exonic circular RNAs as a novel oncogenic driver to promote tumor metastasis in small cell lung Cancer. Clin Cancer Res. 2019:25(4):1302-17.

45. Yin WB, Yan MG, Fang X, Guo JJ, Xiong W, Zhang RP. Circulating circular RNA hsa_circ_0001785 acts as a diagnostic biomarker for breast cancer detection. Clin Chim Acta. 2018;487:363-8.

46. Wang J, Zhang Q, Zhou S, Xu H, Wang D, Feng J, et al. Circular RNA expression in exosomes derived from breast cancer cells and patients. Epigenomics. 2019;11(4):411-21.

47. Jia Q, Ye L, Xu S, Xiao H, Xu S, Shi Z, et al. Circular RNA 0007255 regulates the progression of breast cancer through miR-335-5p/SIX2 axis. Thorac Cancer. 2020;11(3):619-30.

48. Lin J, Cai D, Li W, Yu T, Mao H, Jiang S, et al. Plasma circular RNA panel acts as a novel diagnostic biomarker for colorectal cancer. Clin Biochem. 2019;74:60-8.

49. Pan B, Qin J, Liu X, He B, Wang X, Pan Y, et al. Identification of serum Exosomal hsa-circ-0004771 as a novel diagnostic biomarker of colorectal Cancer. Front Genet. 2019;10:1096.

50. Li Y, Zheng Q, Bao C, Li S, Guo W, Zhao J, et al. Circular RNA is enriched and stable in exosomes: a promising biomarker for cancer diagnosis. Cell Res. 2015;25(8):981-4.

51. Ye DX, Wang SS, Huang Y, Chi P. A 3-circular RNA signature as a noninvasive biomarker for diagnosis of colorectal cancer. Cancer Cell Int. 2019;19:276.

52. Li XN, Wang ZJ, Ye CX, Zhao BC, Huang XX, Yang L. Circular RNA circVAPA is up-regulated and exerts oncogenic properties by sponging miR-101 in colorectal cancer. Biomed Pharmacother. 2019;112:108611.

53. Zhang $X$, Zhao Y, Kong P, Han M, Li B. Expression of circZNF609 is Downregulated in colorectal Cancer tissue and promotes apoptosis in colorectal Cancer cells by Upregulating p53. Med Sci Monit. 2019;25:5977-85.

54. Ji W, Qiu C, Wang M, Mao N, Wu S, Dai Y. Hsa_circ_0001649: a circular RNA and potential novel biomarker for colorectal cancer. Biochem Biophys Res Commun. 2018;497(1):122-6.

55. Li T, Sun X, Chen L. Exosome circ 0044516 promotes prostate cancer cell proliferation and metastasis as a potential biomarker. J Cell Biochem. 2020; 121(3):2118-26.

56. Chen S, Li T, Zhao Q, Xiao B, Guo J. Using circular RNA hsa_circ_0000190 as a new biomarker in the diagnosis of gastric cancer. Clin Chim Acta. 2017; 466:167-71

57. Shao Y, Tao X, Lu R, Zhang H, Ge J, Xiao B, et al. Hsa_circ_0065149 is an Indicator for early gastric Cancer screening and prognosis prediction. Pathol Oncol Res. 2020;26(3):1475-82.

58. Rong D, Lu C, Zhang B, Fu K, Zhao S, Tang W, et al. CircPSMC3 suppresses the proliferation and metastasis of gastric cancer by acting as a competitive endogenous RNA through sponging miR-296-5p. Mol Cancer. 2019;18(1):25.

59. Lu J, Zhang PY, Xie JW, Wang JB, Lin JX, Chen QY, et al. Hsa_circ_0000467 promotes cancer progression and serves as a diagnostic and prognostic biomarker for gastric cancer. J Clin Lab Anal. 2019;33(3):e22726.

60. Chen W, Quan Y, Fan S, Wang H, Liang J, Huang L, et al. Exosometransmitted circular RNA hsa_circ_0051443 suppresses hepatocellular carcinoma progression. Cancer Lett. 2020;475:119-28.

61. Zhu K, Zhan H, Peng Y, Yang L, Gao Q, Jia H, et al. Plasma hsa_circ_0027089 is a diagnostic biomarker for hepatitis B virus-related hepatocellular carcinoma. Carcinogenesis. 2020;41(3):296-302.

62. Yu J, Ding WB, Wang MC, Guo XG, Xu J, Xu QG, et al. Plasma circular RNA panel to diagnose hepatitis B virus-related hepatocellular carcinoma: a large-scale, multicenter study. Int J Cancer. 2020;146(6):1754-63.

63. Qiao GL, Chen L, Jiang WH, Yang C, Yang CM, Song LN, et al. Hsa_cirC_ 0003998 may be used as a new biomarker for the diagnosis and prognosis of hepatocellular carcinoma. Onco Targets Ther. 2019;12:5849-60.

64. Li Z, Zhou Y, Yang G, He S, Qiu X, Zhang L, et al. Using circular RNA SMAR CA5 as a potential novel biomarker for hepatocellular carcinoma. Clin Chim Acta. 2019;492:37-44.

65. Weng Q, Chen M, Li M, Zheng YF, Shao G, Fan W, et al. Global microarray profiling identified hsa_circ_0064428 as a potential immune-associated prognosis biomarker for hepatocellular carcinoma. J Med Genet. 2019;56(1):32-8.

66. Gong Y, Mao J, Wu D, Wang X, Li L, Zhu L, et al. Circ-ZEB1.33 promotes the proliferation of human HCC by sponging miR-200a-3p and upregulating CDK6. Cancer Cell Int. 2018;18:116.
67. Guo W, Zhang J, Zhang D, Cao S, Li G, Zhang S, et al. Polymorphisms and expression pattern of circular RNA circ-ITCH contributes to the carcinogenesis of hepatocellular carcinoma. Oncotarget. 2017;8(29):48169-77.

68. Sun S, Wang W, Luo X, Li Y, Liu B, Li X, et al. Circular RNA circ-ADD3 inhibits hepatocellular carcinoma metastasis through facilitating EZH2 degradation via CDK1-mediated ubiquitination. Am J Cancer Res. 2019;9(8):1695-707.

69. Wang G, Liu W, Zou Y, Wang G, Deng Y, Luo J, et al. Three isoforms of exosomal circPTGR1 promote hepatocellular carcinoma metastasis via the miR449a-MET pathway. EBioMedicine. 2019;40:432-45.

70. Zhang H, Deng T, Ge S, Liu Y, Bai M, Zhu K, et al. Exosome circRNA secreted from adipocytes promotes the growth of hepatocellular carcinoma by targeting deubiquitination-related USP7. Oncogene. 2019;38(15):2844-59.

71. Lei B, Zhou J, Xuan X, Tian Z, Zhang M, Gao W, et al. Circular RNA expression profiles of peripheral blood mononuclear cells in hepatocellular carcinoma patients by sequence analysis. Cancer Med. 2019;8(4):1423-33.

72. Fan L, Cao Q, Liu J, Zhang J, Li B. Circular RNA profiling and its potential for esophageal squamous cell cancer diagnosis and prognosis. Mol Cancer. 2019;18(1):16

73. Zhang Y, Wang Q, Zhu D, Rong J, Shi W, Cao X. Up-regulation of circSMAD7 inhibits tumor proliferation and migration in esophageal squamous cell carcinoma. Biomed Pharmacother. 2019;111:596-601.

74. Wang Q, Zhang Q, Sun H, Tang W, Yang L, Xu Z, et al. Circ-TTC17 promotes proliferation and migration of esophageal squamous cell carcinoma. Dig Dis Sci. 2019;64(3):751-8

75. Hu X, Wu D, He X, Zhao H, He Z, Lin J, et al. circGSK3beta promotes metastasis in esophageal squamous cell carcinoma by augmenting betacatenin signaling. Mol Cancer. 2019;18(1):160.

76. Huang E, Fu J, Yu Q, Xie P, Yang Z, Ji H, et al. CircRNA hsa_circ_0004771 promotes esophageal squamous cell cancer progression via miR-339-5p/ CDC25A axis. Epigenomics. 2020;12(7):587-603.

77. Wang Q, Liu H, Liu Z, Yang L, Zhou J, Cao X, et al. Circ-SLC7A5, a potential prognostic circulating biomarker for detection of ESCC. Cancer Gene. 2020; 240:33-9.

78. Tang X, Liu S, Ding Y, Guo C, Guo J, Hua K, et al. Serum circular FoxO3a serves as a novel prognostic biomarker in squamous cervical Cancer. Cancer Manag Res. 2020;12:2531-40.

79. Wu G, Zhou W, Pan X, Sun Z, Sun Y, Xu H, et al. Circular RNA profiling reveals Exosomal circ_0006156 as a novel biomarker in papillary thyroid Cancer. Mol Ther Nucleic Acids. 2020;19:1134-44.

80. Xu ZQ, Yang MG, Liu HJ, Su CQ. Circular RNA hsa_circ_0003221 (circPTK2) promotes the proliferation and migration of bladder cancer cells. J Cell Biochem. 2018;119(4):3317-25.

81. Chi BJ, Zhao DM, Liu L, Yin XZ, Wang FF, Bi S, et al. Downregulation of hsa_ circ_0000285 serves as a prognostic biomarker for bladder cancer and is involved in cisplatin resistance. Neoplasma. 2019:66(2):197-202.

82. Chen $X$, Chen RX, Wei WS, Li YH, Feng ZH, Tan L, et al. PRMT5 circular RNA promotes metastasis of Urothelial carcinoma of the bladder through sponging miR-30c to induce epithelial-Mesenchymal transition. Clin Cancer Res. 2018;24(24):6319-30.

83. Hu Y, Zhu Y, Zhang W, Lang J, Ning L. Utility of plasma circBNC2 as a diagnostic biomarker in epithelial ovarian Cancer. Onco Targets Ther. 2019; 12:9715-23

84. Wang W, Wang J, Zhang $X$, Liu G. Serum circSETDB1 is a promising biomarker for predicting response to platinum-taxane-combined chemotherapy and relapse in high-grade serous ovarian cancer. Onco Targets Ther. 2019;12:7451-7.

85. Zhao Z, Ji M, Wang Q, He N, Li Y. Circular RNA Cdr1as Upregulates SCAl to suppress Cisplatin resistance in ovarian Cancer via miR-1270 suppression. Mol Ther Nucleic Acids. 2019;18:24-33.

86. Kun-Peng Z, Chun-Lin Z, Jian-Ping H, Lei Z. A novel circulating hsa_circ_ 0081001 act as a potential biomarker for diagnosis and prognosis of osteosarcoma. Int J Biol Sci. 2018;14(11):1513-20.

87. Zhu K, Niu L, Wang J, Wang Y, Zhou J, Wang F, et al. Circular RNA hsa_circ 0000885 levels are increased in tissue and serum samples from patients with osteosarcoma. Med Sci Monit. 2019;25:1499-505.

88. Yang F, Liu DY, Guo JT, Ge N, Zhu P, Liu X, et al. Circular RNA circ-LDLRAD3 as a biomarker in diagnosis of pancreatic cancer. World J Gastroenterol. 2017;23(47):8345-54.

89. Shuai M, Hong J, Huang D, Zhang X, Tian Y. Upregulation of circRNA_ 0000285 serves as a prognostic biomarker for nasopharyngeal carcinoma and is involved in radiosensitivity. Oncol Lett. 2018;16(5):6495-501. 
90. Xu H, Gong Z, Shen Y, Fang Y, Zhong S. Circular RNA expression in extracellular vesicles isolated from serum of patients with endometrial cancer. Epigenomics. 2018;10(2):187-97.

91. Louis C, Desoteux M, Coulouarn C. Exosomal circRNAs: new players in the field of cholangiocarcinoma. Clin Sci (Lond). 2019;133(21):2239-44.

92. Wang R, Zhang S, Chen X, Li N, Li J, Jia R, et al. CircNT5E acts as a sponge of miR-422a to promote Glioblastoma tumorigenesis. Cancer Res. 2018; 78(17):4812-25.

93. Granados-Riveron JT, Aquino-Jarquin G. The complexity of the translation ability of circRNAs. Biochim Biophys Acta. 2016;1859(10):1245-51.

94. Li Z, Huang C, Bao C, Chen L, Lin M, Wang X, et al. Corrigendum: exonintron circular RNAs regulate transcription in the nucleus. Nat Struct Mol Biol. 2017;24(2):194.

95. Qian L, Yu S, Chen Z, Meng Z, Huang S, Wang P. The emerging role of circRNAs and their clinical significance in human cancers. Biochim Biophys Acta Rev Cancer. 2018;1870(2):247-60.

96. Liang ZZ, Guo C, Zou MM, Meng P, Zhang TT. circRNA-miRNA-mRNA regulatory network in human lung cancer: an update. Cancer Cell Int. 2020; 20:173.

97. Yin Y, Long J, He Q, Li Y, Liao Y, He P, et al. Emerging roles of circRNA in formation and progression of cancer. J Cancer. 2019;10(21):5015-21.

98. Zhang HD, Jiang LH, Sun DW, Hou JC, Ji ZL. CircRNA: a novel type of biomarker for cancer. Breast Cancer. 2018;25(1):1-7.

99. Wang Y, Liu J, Ma J, Sun T, Zhou Q, Wang W, et al. Exosomal circRNAs: biogenesis, effect and application in human diseases. Mol Cancer. 2019; 18(1):116.

\section{Publisher's Note}

Springer Nature remains neutral with regard to jurisdictional claims in published maps and institutional affiliations.

Ready to submit your research? Choose BMC and benefit from:

- fast, convenient online submission

- thorough peer review by experienced researchers in your field

- rapid publication on acceptance

- support for research data, including large and complex data types

- gold Open Access which fosters wider collaboration and increased citations

- maximum visibility for your research: over $100 \mathrm{M}$ website views per year

At BMC, research is always in progress.

Learn more biomedcentral.com/submissions 